\title{
HSV-1 stimulation-related protein HSRG1 inhibits viral gene transcriptional elongation by interacting with Cyclin T2
}

\author{
WU WenJuan ${ }^{1,2}$, YU Xian ${ }^{1}$, LI WeiZhong ${ }^{1}$, GUO Lei ${ }^{1}$, LIU LongDing ${ }^{1}$, \\ WANG LiChun ${ }^{1} \&$ LI QiHan ${ }^{1 *}$ \\ ${ }^{1}$ Department of Viral Immunology, Institute of Medical Biology, Chinese Academy of Medical Sciences and \\ Peking Union Medical College, Kunming 650118, China; \\ ${ }^{2}$ Department of Dermatology, First Affiliated Hospital of Kunming Medical College, Kunming 650032, China
}

Received August 25, 2009; accepted February 11, 2010

\begin{abstract}
The protein encoded by HSRG1 (HSV-1 stimulation-related gene 1) is a virally induced protein expressed in HSV-1-infected cells. We have already reported that HSRG1 is capable of interacting with transcriptional regulator proteins. To further analyze the effects of HSRG1 on the regulation of viral gene transcription, we expressed the HSRG1 protein in transfected cells and found that it postpones the proliferation of HSV-1. CAT (chloramphenicol acetyltransferase) assays also revealed that HSRG1 reduces transcription from HSV-1 promoters. Yeast two-hybrid and immunoprecipitation assays indicated that HSRG1 interacts with Cyclin T2, the regulatory subunit of P-TEFb, which is required for transcription elongation by RNA Pol II (RNAP II), and that amino acid residues 1-420 in Cyclin T2 are important for binding with HSRG1. Fluorescence assays suggested that the cellular localizations of those two proteins are influenced by their interaction. Further analyses with CAT assays revealed that HSRG1 inhibits the transcriptional activation by Cyclin T2 of viral promoters. Our results suggested that the inhibitory effects of HSRG1 on viral replication and proliferation are probably induced by its binding to Cyclin T2. Therefore, it is likely that HSRG1 inhibits viral gene transcriptional elongation by interacting with Cyclin T2.
\end{abstract}

herpes simplex virus 1, HSRG1, Cyclin T2, transcriptional regulation

Citation: $\quad$ Wu W J, Yu X, Li W Z, et al. HSV-1 stimulation-related protein HSRG1 inhibits viral gene transcriptional elongation by interacting with Cyclin T2. Sci China Life Sci, 2011, 54: 359-365, doi: 10.1007/s11427-011-4160-3

Herpes simplex virus 1 (HSV-1), a DNA virus possessing both complicated transcriptional mechanisms and genome structure [1], can interact with a latently or lytically infected host [2,3]. Viral components interact with host structural proteins, as well as particular cellular proteins expressed during HSV-1 infection. These interactions can affect the function of cellular components, thereby altering biological processes [4,5]. Existing data demonstrate that cellular expression profiles change in HSV-1-infected cells and have different effects on viral proliferation [6]. Our earlier work focused on analyzing changes in gene expression induced

*Corresponding author (email: liqihan@imbcams.com.cn) by HSV-1 infection in human fibroblasts; HSRG1, encoded by the HSV-1 stimulation-related gene, is one of these proteins [7]. HSRG1 is expressed $2 \mathrm{~h}$ after infection and is classified as a SAND protein family member [8], containing a eukaryotic conserved domain [9] of unknown biological function. Our earlier work also indicated that HSRG1 interacts with the SV40 large T-antigen (LT), altering transcriptional regulation of LT from the SV40 promoter [10]. These results suggested that HSRG1 could interact with transcriptional regulatory proteins. In the present study, we screened candidate cellular proteins that could interact with HSRG1 by a yeast two-hybrid assay, and our results showed that Cyclin T2 was one such protein. Cyclin T2 is an important 
transcriptional regulator. Previous studies have shown that Cyclin T2 binds with CDK9 to form the P-TEFb complex (positive transcription elongation factor $\mathrm{b}, \mathrm{P}-\mathrm{TEFb}$ ), which regulates transcriptional elongation of RNA polymerase II (RNAP II) by phosphorylating transcription-related factors [11]. RNAP II has an important and non-specific role in the gene transcription processes [12], and the interaction of HSRG1 with the Cyclin T2 subunit of the P-TEFb complex indicates that the cellular and viral gene transcriptional process might be affected by this interaction during HSV-1 infection. In the present study, we investigated the possible mechanisms and biological significance of HSRG1 in HSV-1 infection.

\section{Materials and methods}

\subsection{Cells and viruses}

China hamster ovarian cancer cells (CHO-K1) and African green monkey kidney cells (Vero) from our laboratory were grown in F12 supplemented with $10 \%$ (v/v) of fetal bovine serum in T-75 cell culture containers or six-well plates. HSV-1 (F strain) was also provided from our laboratory.

\subsection{Plasmid construction}

Recombination plasmid pcDNA3-CycT2a was constructed by ligating the Cyclin $\mathrm{T} 2$ gene into the eukaryotic expression plasmid, pcDNA3.1 (Invitrogen). The gene encoding Cyclin T2 was obtained by PCR amplification with primers CycT2a-F1 and CycT2a-R1 (Table 1). Eukaryotic expression plasmid pcDNA3-HSRG1, expressing HSRG1, has already been constructed in our laboratory [10]. Recombination plasmid pGADT7-HSRG1 was constructed by ligating the HSRG1 gene (amplified with primers HSRG1-F1 and HSRG1-R1) into the pGADT7 vector (Clontech). Furthermore, pGBKT7-CycT2a, pGBKT7-T2a-250A, pGBKT7T2a-300A, and pGBKT7-T2a-420A were constructed separately by inserting the sequence of Cyclin T2 cDNA, or sequences encoding Cyclin T2 amino acids 1-250, 1-300, or 1-420, into the pGBKT7 vector (Clontech). The Cyclin T2 cDNA and deletion fragments were amplified with primers CycT2-F, CycT2-R; CycT2-F, CycT2-250A-R; CycT2-F, CycT2-300A-R; CycT2-F; and CycT2-420A-R, respectively (Table 1$)$. In addition, pRed-HSRG1 was constructed by inserting the HSRG1 gene (primers CycT2-F2 and CycT2-R2) into the pDsRed-N1 vector (Clontech). Prokaryotic expression plasmid pGEX-4T-CycT2a expressing the Cyclin T2a cDNA was donated by Professor Matija Peterlin. Plasmids pCAT- $\alpha 4$, pCAT-TK, and pCAT-gC plasmids were constructed in this laboratory [13]. All recombinant plasmids were confirmed by enzymatic digestion and DNA sequencing.
Table 1 Primers for PCR amplification

\begin{tabular}{cc}
\hline Primer name & Sequence (5' to 3') \\
\hline HSRG1-F1 & 5'-ATTGAATTCATGGAGCTCGGAGGAGAC-3' \\
HSRG1-R1 & 5'-ACAGAATTCGAGTCCAGTGAACAAGCC-3' \\
HSRG1-F2 & 5'-TATGAATTCGGATGGAGCTCGGAGGA-3' \\
HSRG1-R2 & 5'-TATGGTACCAGTCCAGTGAACAAGCC-3' \\
CycT2-F1 & 5'-ATACTGCAGATATGGCGTCGGGCCGT-3' \\
CycT2-R1 & 5'-ATACTGCAGGACCGTTGGTCTCCACT-3' \\
CycT2-F2 & 5'-ATAAAGCTTAATGGCGTCGGGCCGTGG-3' \\
CycT2-R2 & 5'-ATAGGTACCGGACCGTTGGTCTCCACT-3' \\
T2-250A-R & 5'-GCGCTGCAGCCTATTAGGCGTTTTCTC-3' \\
T2-300A-R & 5'-GCACTGCAGACTTGGGTTTGTAGGCAC-3' \\
T2-420A-R & 5'-GCACTGCAGATGGTGTTTACTGCTCCC-3' \\
\hline
\end{tabular}

\subsection{Plaque assay}

Vero cells were transfected with pcDNA3-HSRG1 or pcDNA3, incubated for $24 \mathrm{~h}$ at $37^{\circ} \mathrm{C}$, and then infected with HSV-1 at MOI 0.5 (multiplicity of infection $(\mathrm{MOI})=$ ratio of infectious virus particles to cells) for absorption for $30 \mathrm{~min}$ at $37^{\circ} \mathrm{C}$. Viruses were harvested at $24 \mathrm{~h}$ and the virus titer was tested by a virus plaque assay and counting plaque forming units (PFU) $\mathrm{mL}^{-1}$ of samples. The results were presented as mean values obtained from three independent experiments.

\subsection{Yeast two-hybrid assay}

Y187 yeast cells transformed with the recombinant plasmid pGADT7-HSRG1 were fused with AH109 yeast cells transformed with recombinant plasmid pGBKT7-CycT2a or pGBKT7-T2a-250A/300A/420A in accord with standard protocols (http://www.clontech.com/products/detail.asp? product_id=206489\&tabno=6). The resulting clones were inoculated into two volumes of YPDA medium for incubation for $20-24 \mathrm{~h}$ at $30^{\circ} \mathrm{C}$. The fused culture was inoculated onto SD/-Leu-Trp plates for incubation for one week at $30^{\circ} \mathrm{C}$. Colonies were inoculated onto SD/-His-Leu-Trp and $\mathrm{SD}$-Ade-His-Leu-Trp plates and incubated for one week at $30^{\circ} \mathrm{C}$, and positive clones were screened.

\section{$1.5 \beta$-galactosidase $(\beta$-gal) activity detection}

Clones were picked and inoculated into $5 \mathrm{~mL}$ SD/-Leu-Trp liquid medium with shaking $\left(200 \mathrm{r} \mathrm{min}^{-1}\right)$ overnight at $30^{\circ} \mathrm{C}$ and then trans-inoculated into YPDA medium with continued shaking for $3-5 \mathrm{~h}$ at $30^{\circ} \mathrm{C}$. $A_{600}$ values were recorded. Yeast cells were collected when the $A_{600}$ value reached 0.5-0.8. Samples were mixed with $4 \mathrm{mg} \mathrm{mL} \mathrm{m}^{-1}$ ortho-Nitrophenyl- $\beta$-galactoside (ONPG) and incubated at $30^{\circ} \mathrm{C}$ until the solution turned a yellow color. The reaction time was recorded. The values for $420 \mathrm{~nm}$ light absorption were recorded using a spectrophotometer after removing cell debris. $\beta$-D-galactosidase content was calculated by the following formula: $\beta$-D-galactosidase units $=1000 \times A_{420} / \mathrm{t} \times$ $5 \times A_{600}$ (where $A_{600}$ is equal to the $A_{600}$ absorption value of 1 
$\mathrm{mL}$ culture and $t$ is the reaction time). The experiment was repeated three times with positive (pGBK-p53+pACT-LT) and negative (pGBK-Lam+pACT-LT) controls.

\subsection{Cell transfection}

CHO cells, grown in six-well plates to $90 \%$ confluence, were transfected using Lipofectamine 2000 (Invitrogen) and the appropriate plasmid, according to the manufacturer's instructions (http://tools.invitrogen.com/content/sfs/manuals/ lf $2 \mathrm{k} \_r$ reverse_transfection_web.pdf).

\subsection{Preparation of Cyclin T2 antibodies}

The E. coli BL21 bacterial strain was transfected with the pGEX-4T-CycT2a plasmid expressing the GST-CycT2a fusion protein following IPTG induction. The purified protein was obtained by excising an SDS-PAGE gel band that contained a protein with the corresponding molecular weight and electroeluting the protein. The same volumes of the purified GST-CycT2a fusion protein and complete Freund's adjuvant were emulsified and mixed for multiple abdominal subcutaneous injections into Kunming mice. The mice were boosted with incomplete Freund's adjuvant emulsions at week 2 and 4. Blood was taken from the eyeball two weeks after the 3rd immunization for preparation of Cyclin $\mathrm{T} 2$ antiserum.

\subsection{Immunoprecipitation and Western blotting}

Vero cells incubated for $36 \mathrm{~h}$ after co-transfection with pcDNA3-HSRG1 and pcDNA3-CycT2a plasmids were rinsed with PBS three times, scraped, treated with RIPA lytic solution $\left(150 \mathrm{mmol} \mathrm{L}^{-1} \mathrm{NaCl}, 1 \% \mathrm{NP}-40,0.5 \%\right.$ sodium deoxycholate, $0.1 \% \mathrm{SDS}, 50 \mathrm{mmol} \mathrm{L}{ }^{-1}$ Tris $\cdot \mathrm{HCl}, \mathrm{pH}$ 7.5) and incubated in an ice bath for $30 \mathrm{~min}$. Protein supernatants were collected by centrifugation. An aliquot of the supernatant was reserved as an input sample. Immunoprecipitation of HSRG1 and Cyclin T2 was carried out according to standard procedures. Antibodies against HSRG1 were added to the remaining lysate supernatants and incubated for $2 \mathrm{~h}$ at $37^{\circ} \mathrm{C}$. Immune complexes were precipitated by incubation with Protein A agarose (Beyotiome). Following incubation, tubes were centrifuged and the beads were washed in lysis buffer. Equal volumes of protein precipitant were subjected to $10 \%$ SDS-PAGE and transferred to a PVDF membrane followed by blocking with $5 \%$ BSA-TTBS. Western blot analysis was performed using HSRG1 or Cyclin T2 antisera as primary antibodies and sheep anti-mouse IgG-HRP as the secondary antibody for DAB reaction.

\subsection{Chloramphenicol acetyltransferase (CAT) activity analysis}

$\mathrm{CHO}$ cells were transfected and incubated for $48 \mathrm{~h}$ at $37^{\circ} \mathrm{C}$.
The CAT and $\beta$-galactosidase activities in $\mathrm{CHO}$ cell extracts in each group were detected by the CAT enzyme assay system and the $\beta$-gal enzyme assay system, respectively, in accord with standard protocols (Promega). $\beta$-gal activity values were used to normalize CAT activity values. The relative CAT activity in each experimental group was calculated by defining the CAT activity values detected by co-transfection of pcDNA3 plasmid and CAT reporter gene expression plasmids as 100 . The results were presented as the mean values obtained from three independent experiments.

\subsection{Analysis of protein localization}

Vero cells incubated for $40 \mathrm{~h}$ after co-transfection with pRed-HSRG1 and pEGFP-CycT2 plasmids were examined with an immunofluorescent microscope. Photographs were taken and merged to analyze the locations of fluorescent fusion proteins.

\subsection{Statistical analysis}

All data were statistically analyzed according to standard protocols. Results are expressed as means \pm SD. Statistical significance was determined using two-way analysis of variance and the Student's $t$-test using SPSS 11.0 software. $P$-values $<0.05$ were considered statistically significant.

\section{Results}

\subsection{Expression of HSRG1 delays the proliferation of HSV-1}

HSRG1 is a host protein expressed during HSV-1 infection. To analyze the effect of HSRG1 on HSV-1 proliferation, Western blot analysis was performed on extracts from transfected Vero cells using HSRG1 antisera as the primary antibody. The results showed that HSRG1 was expressed in pcDNA3-HSRG1-transfected cells (Figure 1A). Plaque assays were then used to analyze the effects of HSRG1 on virus titer. Vero cells transfected with pcDNA3-HSRG1 or pcDNA3 were infected with HSV-1 at an MOI of 0.5, $24 \mathrm{~h}$ after transfection. Viruses were harvested at 12 or $24 \mathrm{~h}$. The results showed that virus titers in cells transfected with pcDNA3-HSRG1 were lower than in the control group, indicating that high expression of HSRG1 delayed the proliferation of HSV-1 (Figure 1B).

\subsection{HSRG1 inhibits transcriptional elongation effi- ciency on HSV-1 $\alpha 4, T K$, and $g C$ promoters}

To analyze the effect of HSRG1 on virus proliferation, CAT assays were carried out to detect the transcriptional regulatory effect of HSRG1 on HSV-1 gene promoters. The eu- 

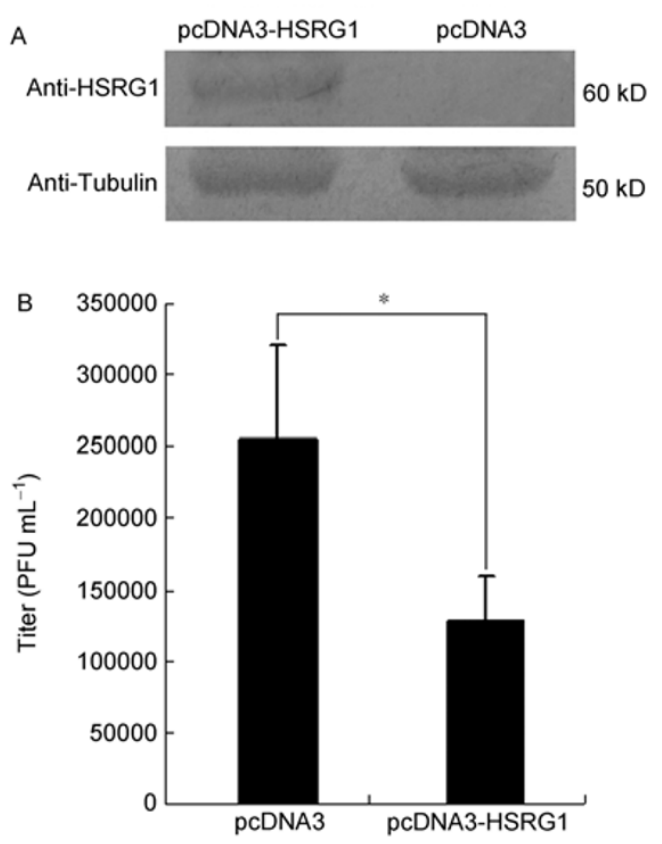

Figure 1 Expression of HSRG1 protein in Vero cells and its effect on HSV-1 proliferation. A, Vero cells transfected with pcDNA3 or pcDNA3HSRG1 were harvested $24 \mathrm{~h}$ after transfection, and the expression of HSRG1 was detected by Western blotting using anti-HSRG1 antiserum. Tubulin was detected by anti-Tubulin antiserum as a loading control. B, Vero cells were transfected with pcDNA3-HSRG1 or pcDNA3, incubated at $37^{\circ} \mathrm{C}$ for $24 \mathrm{~h}$, and infected with $\mathrm{HSV}-1$ at MOI 0.5 . Virus was harvested at $36 \mathrm{~h}$. Virus titers were tested by a virus plaque assay. * indicates $P<0.05$.

karyotic expression plasmid pcDNA3-HSRG1 was cotransfected with reporter plasmids pCAT- $\alpha 4$, pCAT-TK, or pCAT-gC into CHO-K1 cells. CAT activity was measured to analyze the effects of HSRG1 on different viral gene promoters. The results showed that HSRG1 protein had different inhibitory effects on HSV-1 immediate-early, early, and late gene transcription (Figure 2) and that this effect was somewhat dose-dependent.

\subsection{HSRG1 interacts with Cyclin T2a protein}

To analyze proteins interacting with HSRG1, we selected a number of possible candidate proteins using yeast twohybrid experiments. A human liver cell cDNA library was screened using HSRG1 as the bait protein (Table 2). Cyclin $\mathrm{T} 2$ was one of the candidate proteins. Cyclin $\mathrm{T} 2$ contains several conserved structural domains, including an $\mathrm{N}$-terminal Cyclin box, a histidine rich region, and a leucine rich region. These are important for the formation of the $\mathrm{P}-\mathrm{TEFb}$ complex by Cyclin T2/CDK9 dimerization and for binding with RNAP II. In this study, recombinant plasmids pGBKT7-CycT2a, pGBKT7-T2a-250A, pGBKT7-T2a-300A, and pGBKT7-T2a-420A were constructed by ligating the sequences of Cyclin T2 and a series of truncated fragments of its N-terminal 250, 300, and 420 amino acids (Figure 3A) into the pGBKT7 vector. AH109 yeast cells transfected

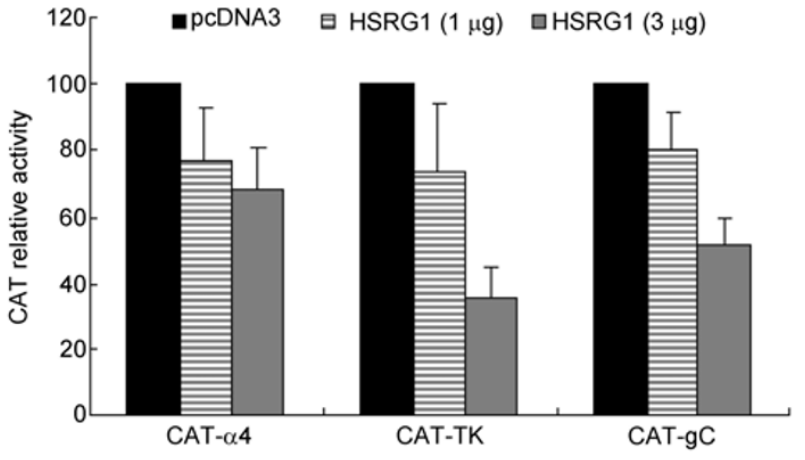

Figure 2 Dose-response curve for transcriptional repression of HSRG1 on HSV-1 promoters. $1 \mathrm{mg}$ of CAT reporter plasmid CAT- $\alpha 4$, CAT-TK, or CAT-gC was co-transfected into Vero cells with different amounts of pcDNA3-HSRG1. The amount of total transfected DNA was kept constant by addition of pcDNA3 in every well. Cells were harvested at $48 \mathrm{~h}$. The relative CAT activity values in each experimental group were calculated by assigning a value of 100 to CAT activity values detected by transfecting

$1 \mu \mathrm{g}$ CAT reporter gene expression plasmids and $1 \mu \mathrm{g}$ pcDNA3.

Table 2 Candidate genes encoding proteins interacting with HSRG1

\begin{tabular}{ccc}
\hline No. & Gene & Accession No. \\
\hline 11 & cyclinT2 & NM-00124 \\
43 & flotillin1 & BC-001146 \\
70 & SR-A1 & NM-021228 \\
112 & unknown protein & AW-307598 \\
145 & SV40 large T antigen & NP-043127 \\
\hline
\end{tabular}

with these recombinant plasmids were fused with Y187 yeast cells transfected with pGADT7-HSRG1. The results suggested that potential interactions existed between HSRG1 and multiple regions of Cyclin T2 (Figure 3B). $\beta$-galactosidase activity of yeast clones grown on SD/-AdeHis-Leu-Trp plates was detected, suggesting that amino acids 1-250 and 300-420 of Cyclin T2a have significant contributions to protein interactions (Figure 3D).

Yeast two-hybrid experiments showed that the physical interaction of HSRG1 and Cyclin T2 is mediated by amino acids 1-250 and 300-420 of Cyclin T2. The former contains the cyclin box, a conserved domain that could bind to CDK9. To further verify the relationship between these two proteins in cells, immunoprecipitation was performed using anti-HSRG1 antiserum in Vero cells transfected with pcDNA3-HSRG1 and pcDNA3-CycT2a. Cyclin T2 complexed with HSRG1 was detected by co-immunoprecipitation, followed by Western blotting with anti-Cyclin T2 antiserum (Figure 3C). These results confirmed the interaction between HSRG1 and Cyclin T2. Additionally, a yeast two-hybrid $\beta$-gal assay also revealed the amino acid regions responsible for the interaction between HSRG1 and Cyclin T2 (Figure 3D).

\subsection{HSRG1 protein colocalizes with Cyclin T2 protein in living cells}

To verify the interaction between HSRG1 and Cyclin T2 in 
A

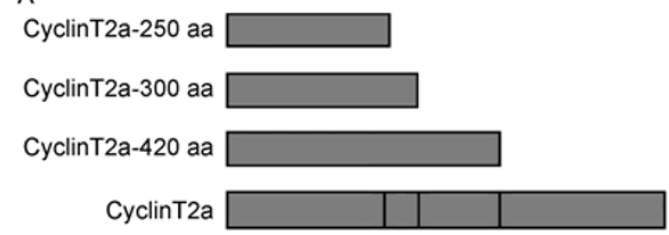

B

\begin{tabular}{|l|c|c|c|}
\hline \multicolumn{1}{|c|}{ Plasmids transfected } & DDO & TDO & QDO \\
\hline AD-HSRG1+BD-CycT2a 250 & +++ & +++ & +++ \\
\hline AD-HSRG1+BD-CycT2a 300 & ++ & ++ & + \\
\hline AD-HSRG1+BD-CycT2a 420 & +++ & +++ & +++ \\
\hline AD-HSRG1+BD-CycT2a & +++ & +++ & ++ \\
\hline
\end{tabular}

C

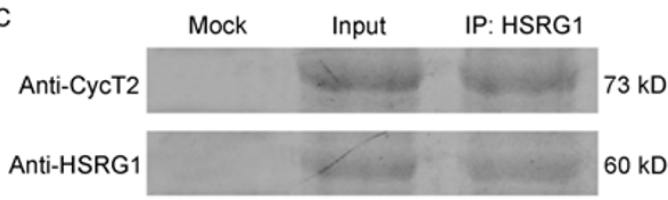

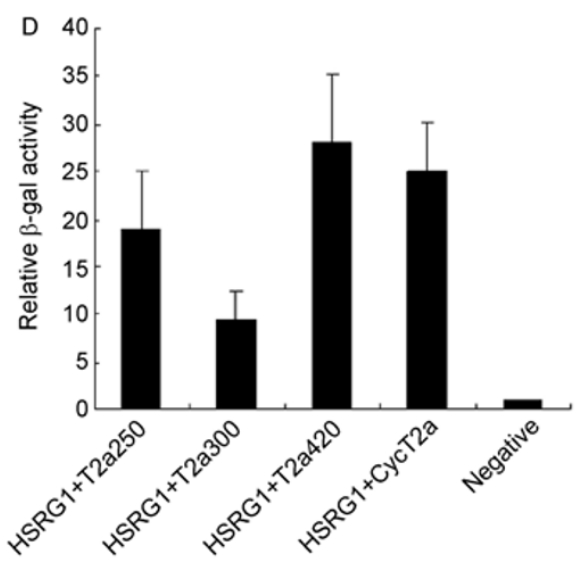

Figure 3 Protein interactions between HSRG1 and Cyclin T2a. A, Schematic for Cyclin T2a and a series of truncated fragments. A series of recombination plasmids was constructed by separately inserting the sequence of Cyclin T2 cDNA, or sequences encoding amino acids 1-250, 1-300, and 1-420 of Cycling T2, into the pGBKT7 vector. These plasmids were used in yeast two-hybrid assays. B, Table of Scores for yeast growth on SD/-Leu-Trp (DDO), SD/-His-Leu-Trp (TDO), and SD/-Ade-His-Leu-Trp (QDO) plates. Y187 yeast cells transformed with pGADT7-HSRG1 were fused with AH109 yeast cells transformed with pGBKT7-CycT2a or pGBKT7-T2a-250A/300A/420A. Plate mating culture and controls on appropriate SD selection media are shown. Plates were incubated at $30^{\circ} \mathrm{C}$ until colonies appeared and subsequently scored for growth. C, Immunoprecipitation of HSRG1 and Cyclin T2a. Vero cells grown in 10-cm dishes to $80 \%$ confluence were transfected with pcDNA3-HSRG1 and pcDNA3-CycT2a. Immunoprecipitation was performed in Vero cells $40 \mathrm{~h}$ after transfection using HSRG1 antiserum. Western blots were carried out using HSRG1 or Cyclin T2a antisera. Mock, lytic supernatants from untransfected Vero cells; Input, Part of the transfected cells lytic supernatant was reserved as an input sample. D, $\beta$-galactosidase activity. Clones were isolated and inoculated in SD/-Leu-Trp liquid medium. Yeast cells were collected when the $A_{600}$ value reached $0.5-0.8$. Lysate supernatants of yeast cells were incubated with ONPG and the reaction was terminated when the solution turned yellow. Reaction time and $A_{420}$ values were recorded. $\beta$-D-galactosidase activity units were calculated by the following formula: $\beta$-D-galactosidase units $=1000 \times A_{420} / t \times 5 \times A_{600}$ (where $A_{600}$ is equal to the $A_{600}$ absorption value of 1 mL culture and $t$ is the reaction time). The results are presented as mean values obtained from three independent experiments.

living cells, recombinant plasmids pRed-HSRG1 and pEGFP-T2a were constructed and transfected into Vero cells. HSRG1 was expressed as a fusion protein with red fluorescent protein, and Cyclin T2a was expressed as a fusion protein with green fluorescent protein. The distribution of fluorescent proteins was observed at $48 \mathrm{~h}$. As shown in Figure 4, Cyclin T2a-GFP localized in the nucleus and HSRG1-RFP localized in the cytoplasm when plasmids pRed-HSRG1 or pEGFP-T2a were transfected alone (Figure 4A). However, colocalization of both signals was observed in both the nucleus and the cytoplasm when the two plasmids were co-transfected into Vero cells (Figure 4B). Taken together, these experiments suggested that the HSRG1 protein interacts with Cyclin T2 protein in cells, and that the cellular localizations of these two proteins were changed to some extent by these interactions.

\subsection{HSRG1 can block the promotion of Cyclin T2-me- diated transcriptional activation of $\mathrm{HSV}-1$ virus pro- moters}

Our experiments indicated that HSRG1 could interact with
Cyclin T2. Furthermore, CAT assays showed inhibitory effects of HSRG1 on several viral gene promoters. Considering the biological significance of Cyclin $\mathrm{T} 2$ in the $\mathrm{P}-\mathrm{TEFb}$ transcriptional elongation factor complex, CAT reporter gene activity was assessed in CHO-K1 cells co-transfected with pcDNA3-HSRG1 or pcDNA3-CycT2a together with CAT reporter plasmids to analyze the effect of HSRG1 on transcriptional promotion by Cyclin $\mathrm{T} 2$. The results revealed that transcriptional activation of immediate-early, early, and late HSV-1 promoters was upregulated in cells transfected with pcDNA3-CycT2a, whereas, in cells co-transfected with pcDNA3-HSRG1 and pcDNA3-CycT2a, the presence of HSRG1 was able to block Cyclin T2-mediated transcriptional activation of different viral promoters (Figure 5A-C). Thus, HSRG1, a protein expressed in the early stages of HSV-1 infection, binds with Cyclin T2, the regulatory subunit of the $\mathrm{P}-\mathrm{TEFb}$ transcriptional elongation complex to downregulate Cyclin T2-mediated activation of viral promoters during HSV-1 infection. It is likely that HSRG1 functions to inhibit viral proliferation by inhibiting activation of viral promoters. 

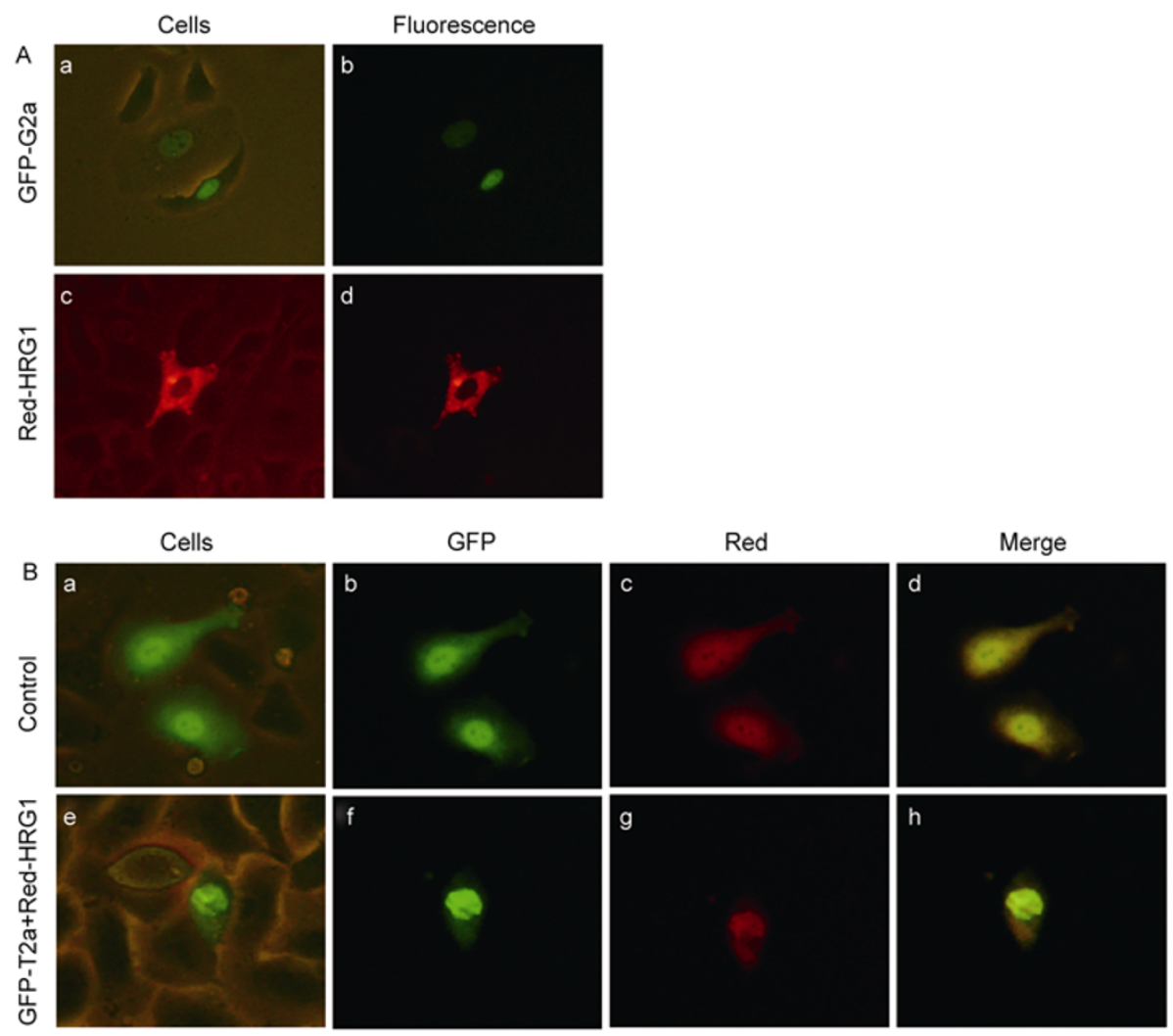

Figure 4 Fluorescent colocalization of Cyclin T2a and HSRG1. A, Cellular localization of Cyclin T2a or HSRG1. Green fluorescence indicates the cellular location of a Cyclin T2a fusion protein expressed by pEGFP-CycT2a (abbreviated as GFP-T2a), and red fluorescence indicates the location of a HSRG1 fusion protein expressed by pDsRed-HSRG1 (abbreviated as Red-HSRG1). B, Cyclin T2a colocalizes with HSRG1. a-d, Vero cells transfected with pEGFP and pDsRed plasmids. e-h, Vero cells transfected with pEGFP-CycT2a and pDsRed-HSRG1 plasmids.

\section{Discussion}

Proteins induced by viral infection have been intensively studied $[6,7,10]$ and the functions of these proteins have been gradually clarified. HSRG1, belonging to the conserved SAND protein family, is one of the proteins upregulated in early stages of HSV-1 infection. As a member of the SAND family, it is important to understand the functions of HSRG1. We previously found that HSRG1 specifically interacts with amino acids $132-450$ of the SV40 large $\mathrm{T}$ antigen, and that transcriptional activity of SV40 promoters activated by large $\mathrm{T}$ was inhibited by HSRG1 [10]. We suggested that HSRG1 might have a role in cellular transcriptional regulation. The results presented here indicate that upregulation of HSRG1 inhibits proliferation of HSV-1. In addition, CAT assays found that HSRG1 inhibits transcriptional elongation efficiency on the HSV-1 $\alpha 4, T K$, and $g C$ promoters and that this effect was somewhat dosedependent. These results suggested that HSRG1 has an important role in viral gene transcription.

Transcription in eukaryotic cells involves three major steps: initiation, elongation, and termination. After successful initiation of RNA synthesis, RNA pol II can pause, be arrested, pass through terminator sequences, or terminate transcription. The post-initiation processivity of RNA pol II is con- trolled by both negative and positive factors. The P-TEFb complex is composed of a catalytic subunit (cyclin-dependent kinase CDK9) and a subunit, Cyclin T2. Cyclin T2 is proposed to facilitate the transition from abortive to productive elongation by phosphorylating the C-terminal domain (CTD) of the largest subunit of RNA pol II [12]. Cyclin T2 has two forms, termed T2a and T2b, which likely arise by alternative splicing of the primary transcript. Although they share the N-terminus, they have different carboxy termini [14], both of which can bind with CDK9 to form a P-TEFb complex to promote transcriptional efficiency by productive transcriptional elongation. Like all Cyclin $\mathrm{T}$ proteins, Cyclin T2a contains two conserved domains: the Cyclin box, formed by 250 amino acids that can bind with CDK9, and the histidine-rich stretch, which binds to the CTD of RNAP II and direct the transcriptional activity of the P-TEFb complex. In addition to these two domains, Kurosu et al. [15] found a leucine-rich region next to the cyclin boxes in Cyclin T2, which are responsible for maintaining appreciable transcriptional activity and binding RNAP II via its CTD. It has been suggested that Cyclin T2a exerts its positive effect by interacting with CDK9 and RNAP II via different domains to shorten their spatial distance, promoting the phosphorylation of the largest subunit CTD of RNA pol II by CDK9. In this study, a 

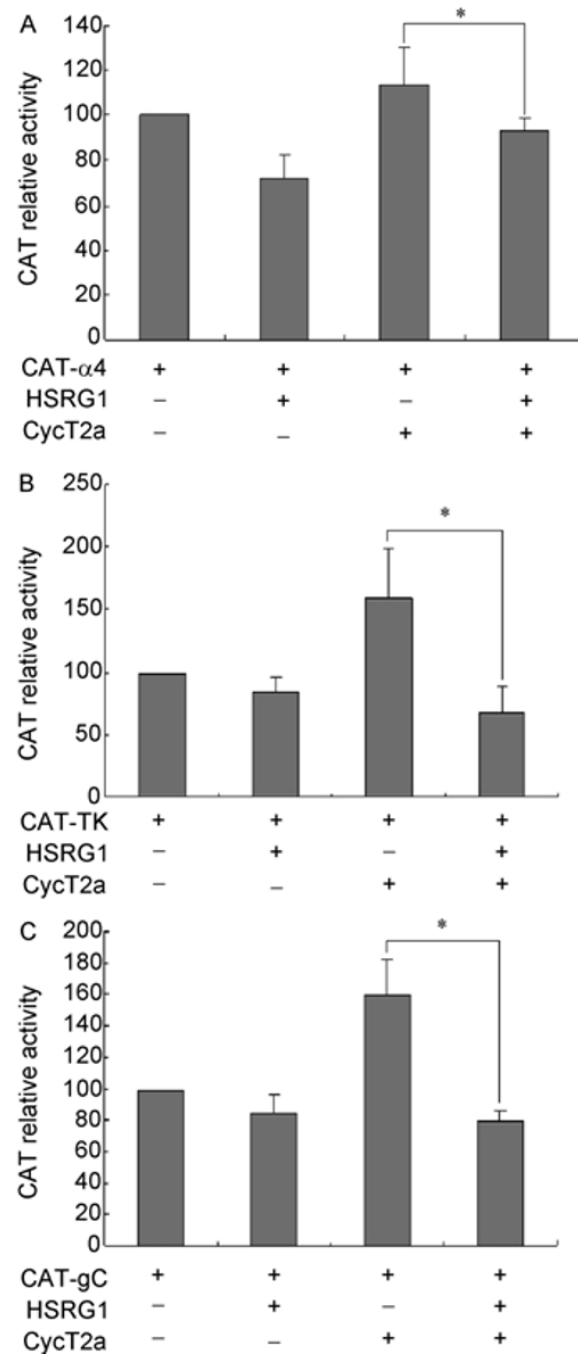

Figure 5 HSRG1 inhibits Cyclin T2-mediated transcriptional activation of HSV-1 virus promoters. A-C, HSRG1 inhibits Cyclin T2-mediated activation of $\alpha 4, T K$, or $g C$ promoters. $1 \mathrm{mg}$ of reporter plasmid CAT- $\alpha 4$ (Figure 5A), CAT-TK (Figure 5B), or CAT-gC (Figure 5C) was co-transfected into $\mathrm{CHO}$ cells with different combinations of $1 \mu \mathrm{g}$ pcDNA3-HSRG1 or $1 \mu \mathrm{g}$ pcDNA3-CycT2a. The total amount of transfected DNA was kept constant by the addition of pcDNA3 in every well. Samples were harvested $48 \mathrm{~h}$ after transfection. The relative CAT activity in each experimental group was calculated by defining the CAT activity values detected by the combination of pcDNA3 plasmid and CAT reporter gene expression plasmid transfection as $100, n=3$. * indicates $P<0.05$.

yeast two-hybrid analysis showed that multiple regions of Cyclin T2a are important for interaction with HSRG1, including the N-terminal Cyclin box domain. Fluorescent protein localization experiments analyzed the effect of this interaction on the spatial location of these proteins. These results established that HSRG1 and Cyclin T2a localized in the cytoplasm and nucleus separately when plasmids were transfected alone, while the nuclear localization of Cyclin
T2 was partly blocked by HSRG1. Furthermore, some HSRG1 was transferred to the nucleus by Cyclin T2 when the plasmids were co-transfected. Therefore, it is reasonable to speculate that the biological function of Cyclin T2a might be affected by binding with HSRG1. The effect of HSRG1 on transcriptional promotion by Cyclin $\mathrm{T} 2 \mathrm{a}$ was analyzed. The results demonstrated that HSRG1 could reverse the positive transcriptional regulation of Cyclin T2 and could inhibit the expression of multiple HSV-1 genes, to varying degrees. This inhibitory effect could be explained by HSRG1 blocking or depleting Cyclin T2 such that transcriptional efficiency was downregulated because of reduced levels of P-TEFb complexes. Considering the association of HSV-1 infection and high expression of HSRG1, it is likely that HSRG1 constitutes a cellular defense against viruses by limiting viral gene duplication in infected cells.

This work was supported by the National Natural Science Foundation of China (Grant Nos. 30700028, 30670094) and the Doctoral Fund of Ministry of Education of China (Grant No. 0060023008).

1 Weir J P. Regulation of herpes simplex virus gene expression. Gene, 2001, 271: 117-130

2 Shimomura Y. Herpes simplex virus latency, reactivation, and a new antiviral therapy for herpetic keratitis. Nippon Ganka Gakkai Zasshi, 2008, 112: 247-264

3 Minagawa $\mathrm{H}$. Latency and reactivation of human alpha herpesviruses. Nippon Rinsho, 2006, 64, Suppl 3: 192-197

4 Herbst-Kralovetz M, Pyles R. Toll-like receptors, innate immunity and HSV pathogenesis. Herpes, 2006, 13: 37-41

5 Li J F, Liu L D, Ma S H, et al. HTRP-an immediate-early gene product induced by HSV1 infection in human embryo fibroblasts, is involved in cellular co-repressors. J Biochem, 2004, 136: 169-176

6 Li Q H. Cytokines and Immediate Early Genes Reactions. Kunming: Yunnan University Press, 2001. 34-57

7 Dong S, Dong C, Liu L, et al. Identification of a novel human sand family protein in human fibroblasts induced by herpes simplex virus 1 binding. Acta Virol, 2003, 47: 27-32

8 Cottage A, Edwards Y J, Elgar G, et al. A new protein family: From nucleic acid to protein structure and function prediction. Comp Funct Genomics, 2001, 2: 226-235

9 Poteryaev D, Spang A. A role of SAND-family proteins in endocytosis. Biochem Soc Trans, 2005, 33: 606-608

10 Guo H X, Cun W, Liu L D, et al. Protein encoded by HSV-1 stimulation-related gene 1 (HSRG1) interacts with and inhibits SV40 large T antigen. Cell Prolif, 2006, 39: 507-518

11 Marshall N F, Price D H. Purification of P-TEFb, a transcription factor required for the transition into productive elongation. J Biol Chem, 1995, 270: 12335-12338

12 Marshall N F, Peng J, Xie Z, et al. Control of RNA polymerase II elongation potential by a novel carboxyl-terminal domain kinase. J Biol Chem, 1996, 271: 27176-27183

13 Yu X, Li W Z, Liu L D, et al. Functional analysis of transcriptional regulation of herpes simplex virus type 1 tegument protein VP22. Sci China Ser C-Life Sci, 2008, 51: 966-972

14 Peng J, Zhu Y, Milton J T, et al. Identification of multiple cyclin subunits of human P-TEFb. Genes Dev, 1998, 12: 755-762

15 Kurosu T, Zhang F, Peterlin B M. Transcriptional activity and substrate recognition of cyclin T2 from P-TEFb. Gene, 2005, 343: 173-179

Open Access This article is distributed under the terms of the Creative Commons Attribution License which permits any use, distribution, and reproduction in any medium, provided the original author(s) and source are credited. 